\title{
CAEN Educational: Nuclear and Particle Physics Experiments
}

\author{
C. Mattone ${ }^{1}$, M. Antonello ${ }^{2}$, M. Locatelli ${ }^{1}$, V. Arosio ${ }^{2}$, L. Malinverno 2 , S. Lomazzi ${ }^{2}$, R. de Asmundis ${ }^{3}$ \\ ${ }^{I}$ CAEN S.p.A., Via Vetraia 11, I-55049, Viareggio, LU, Italy \\ ${ }^{2}$ Università degli Studi dell'Insubria Via Valleggio, 11, 22100, Como (Italy) \\ ${ }^{3}$ INFN Naples, Complesso Monte S. Angelo, Via Cinthia, 80100, Napoli (Italy)
}

\begin{abstract}
CAEN S.p.A., an important industrial spin-off of the INFN (National Institute for Nuclear Physics), is pleased to present its new activities in the educational field. CAEN brings the experience acquired in almost 40 years of collaboration with the High Energy \& Nuclear Physics community into the university educational laboratories by providing modern physics experiments based on the latest technologies and instrumentation. CAEN has realized different modular Educational Kits, all based on Silicon Photomultipliers (SiPM) state of-the-art light sensors with single photon sensitivity and unprecedented photon number resolving capability. They have proven to be suitable for an increasing number of applications in science and industry. The main goal is to inspire students and guide them towards the analysis and comprehension of different physics phenomena with a series of experiments based on state-of-the art technologies, instruments and methods.
\end{abstract}

\section{INTRODUCTION}

The CAEN Educational Kits are modern and flexible platforms for teaching the fundamentals of statistics, particles detection and nuclear imaging.

The setups are entirely based on a new class of photon sensors: Silicon Photomultipliers (SiPMs) featuring single photon detection sensitivity, high photon detection efficiency, low excess noise factor and extended dynamic range. SiPMs are novel light detectors consisting of a matrix of p-n junctions with a common output and a cell density. Each diode is operated in a limited Geiger-Muller regime in order to achieve very high gains $\left(10^{6}\right.$ level $)$ and to guarantee a homogeneity in the cell-to-cell response [1][2][3][4]. All of the diodes in the matrix are connected in parallel to a single output providing a measurement of the incoming light intensity with a signal proportional to the number of fired cells.

These photosensors are evolving rapidly and it shows great promise for applications in both fundamental and applied science: particle and nuclear physics, biology, environmental science, diagnostics in medicine and industry.

This is the reason why, a few years ago, an R\&D program was started in collaboration with Insubria University (ComoItaly) to design a modular set-up that allows to easily characterize SiPMs and build a platform for a series of experiments aimed for both undergraduate and graduate students in physics and engineering.

A series of exemplary activities have been prepared to address a plurality of topics concerning Poisson statistics, gamma spectrometry, cosmic ray detection and more [5]. The activities address experiments with different difficulty levels in order to target them to the students educational levels, from high school to undergraduate and even Ph.D. courses. Dedicated software is being developed, including a User's Guide, reference papers and, for each topic, several online/offline analysis tools.

This paper introduces the elements of the system and focuses on the basics of the quantum nature of light, on statistical properties of the light pulses and cosmic ray detection as exemplary illustrations of the use of the kit in the laboratory activities during the Conference on Lab Instruction Beyond the First Year (BFY III) [6].

\section{EXPERIMENTAL SETUP}

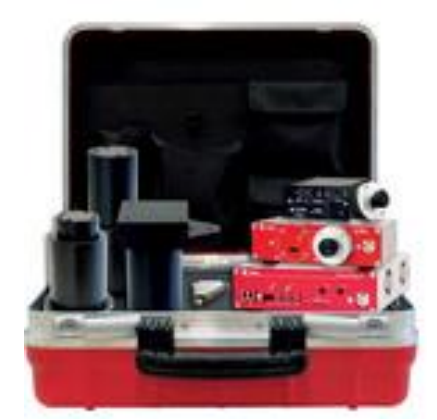

Fig 1: The CAEN educational kit full package.

The Educational Kit - Premium Version, SP5600AN, is the system solution that includes all the components of the other educational kits: SP5600C, SP5600D and SP5600E. The kit can be configured to perform several experiments, covering different Physics fields. The set-up consists of modular plug and play devices USB controlled by a computer. A user friendly Graphical User Interface (GUI) allows the user to easily operate the system and to perform a series of measurements. The CAEN Premium Version kit [7], shown in Fig. 1, consists of: 
- A Power Supply \& Amplification Unit (PSAU, SP5600). The PSAU supplies the bias for the sensors, features a variable amplification factor up to $50 \mathrm{~dB}$ and integrates a feedback circuit to stabilize the sensor gain against temperature variations. Moreover, the PSAU integrates a leading-edge discriminator per channel plus coincidence logic.

- A Desktop Waveform digitizer (DT5720A), with 2 input channels sampled at $250 \mathrm{MS} / \mathrm{s}$ by a 12 bit ADC. The available firmware enables the possibility to perform charge integration and pulse shape discrimination.

- A Ultra-fast LED Driver (SP5601) with a pulse width of $8 \mathrm{~ns}$, tuneable intensity, pulsing frequency internally/externally generated and FC interface to either a clear or a Wave Length Shifting (WLS) fibre. Moreover, the emitted light pulses are at $400 \mathrm{~nm}$ with FWHM of $14 \mathrm{~nm}$. The pulses are characterized by an exponential time distribution of the emitted photons with a rising edge at sub-nanosecond level and a trailing edge with $\tau \sim 5$ ns. The driver also provides a synchronization signal in TTL standard.

- A Scintillating Tile (SP5608) composed of a support with an embedded plastic scintillating tile $\left(48 \times 48 \times 10 \mathrm{~mm}^{3}\right)$, directly coupled to a SiPM $\left(6 \times 6 \mathrm{~mm}^{2}\right)$. The tile is the ideal tool for tests with beta emitting isotopes and cosmic rays. It is provided with paper and aluminium sheets and a source holder in order to perform beta attenuation measurements.

- A Mini-Spectrometer for gamma ray detection (SP5606) composed of a mechanical structure that houses a scintillating crystal $\left(15 \times 6 \times 6 \mathrm{~mm}^{3}\right)$, coupled to a dedicated SiPM $\left(6 \times 6 \mathrm{~mm}^{2}\right)$. Three different crystals are available: CsI, LYSO and BGO. The spectrometer is equipped with a bottom support to allow an easy connection to the SP5600 via the splitter A315, to avoid saturation effects. The spectrometer head was designed to make easy a full range of basic gamma spectrometry educational experiments.

- A SiPM Holder (SP5650C), housing a Hamamatsu MPPC $1.3 \times 1.3 \mathrm{~mm}^{2}$ model S13360-1350CS. The mechanical structure of the holder allows an easy coupling of the holder itself with the PSAU.

- The Gamma absorption tool (SP5607) allows the performance of gamma attenuation measurements. It is a modular tool and its design allows an easy connection to the SP5606 bottom support. The SP5607 is composed of spacers, Aluminium and PMMA absorbers (one $4 \mathrm{~mm}$ thick, five $10 \mathrm{~mm}$ thick).

The different building blocks of the kit can be assembled in a customized configuration, according to the specific application and the user's requirements, allowing maximum flexibility for the different activities.

\section{EDUCATIONAL ACTIVITIES}

Exploring the quantum nature of phenomena is one of the most exiting experiences a physics student can have, together with the detection of cosmic rays, energetic and subatomic particles constantly bombard the Earth's atmosphere from all directions.

Radioactivity is around us and getting to know it experimentally is essential for physics students. When an unstable nucleus decays in a cascade leading to a stable nuclide, it emits either an $\alpha, \beta$ or $\gamma$ particle or a combination of them. The spectroscopy of the emitted $\gamma$ or $\beta$ rays is instrumental for understanding the mechanism of their interaction with matter, the fundamentals about detection and the underlying nuclear physics. Moreover, it is relevant in basic and applied fields of science and technology, from nuclear to medical physics, from archaeometry to homeland security. Beta spectroscopy, furthermore, introduces the student into the field of special relativity and the weak interactions of radioactive decays.

All the proposed experiments have their own step by step guide that includes a detailed description to perform the data analysis of the physical process. The experiments address the essence of the phenomenon as well as exemplary illustrations of their use in medical imaging and industry, complemented by basic and advanced statistical exercises.

In this paper section, a brief description of the two application fields is presented: photon detection and cosmic rays.

\section{A. Photon Detection}

In the XVII century the concept of wave-particle duality was developed, starting from the wave nature of light postulated by Huygens to the Einstein Photoelectric Effect, which postulates light quanta existence, late in the XIX century and in the XX century. A basic principle of quantum mechanics is complementarity: each quantum-mechanical object has both wave-like and particle-like properties. With this approach, the photon is at once a wave and particle, but they can never be observed simultaneously in the same experiment, not even if the uncertainty principle is successfully bypassed.

Due to the educational kit (Fig.2) driven by a dedicated Control software, the exploration of the quantum nature of light thanks to bunches of photons emitted in a few nanoseconds by an ultra-fast LED and sensed by a Silicon Photomultiplier is allowed. 


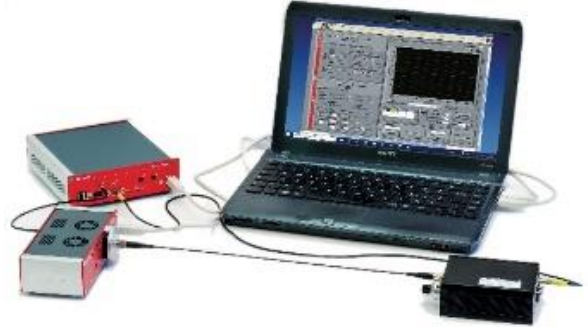

Fig 2: CAEN Educational Photon kit.

The typical SiPM response to a light pulse (Fig.3) is characterized by multiple traces, each one corresponding to different numbers of fired cells, proportional to the number of impinging photons. Each cell of the SiPM is subject to the high electric field in the depletion zone, initial charge carriers generated by an absorbed photon trigger an exponential charge multiplication by impact ionization, till when the current across the quenching resistance induces a drop in the operating voltage, stopping the process. Each spectrum peak corresponds to different number of cells fired at the same time. Because of the high gain compared to the noise level, the traces are well separated, providing a photon number resolved detection of the light field.

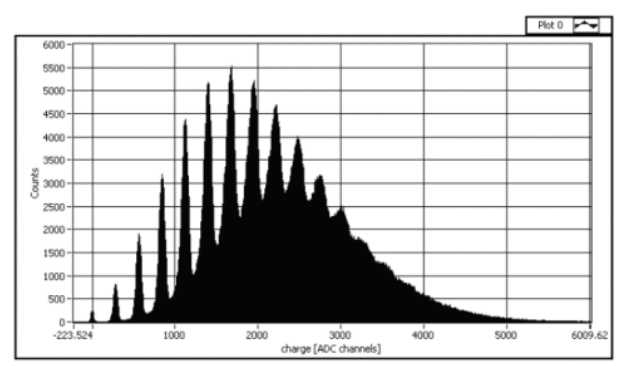

Fig.3: Spectrum of the photons emitted by a LED Driver and detected by a Silicon Photomultiplier.

In the spectrum of the SiPM response to a light pulse, every entry corresponds to the digitized released charge, measured integrating the electrical current spike during a pre-defined time interval. The peaks correspond to different number of cells fired at the same time by incoming photons. This class of detectors can count the number of impacting photons, shot by shot, allowing the observation that the light is composed by photons. Moreover, the SiPM measures the light intensity simply by the number of fired cells and it is possible to investigate the statistical properties of light.

The spontaneous emission of light results from random decays of excited atoms and it is expected to follow the Poisson distribution (Fig.4). The features of SiPMs allow the student to apply basic skills in probability and statistics to the analysis of light quanta of the spectrum coming from the SiPM response to the light pulses.
In SiPM the homogeneity of the response is quite high, however, since fired cells are randomly distributed in the detector sensitive area residual differences in the gain become evident broadening the peak. A key point in the analysis technique was the estimation of the area underneath every peak, essential to reconstruct the probability density function of the emitted number of photons per pulse. An easy procedure is to consider each peak as a gaussian.
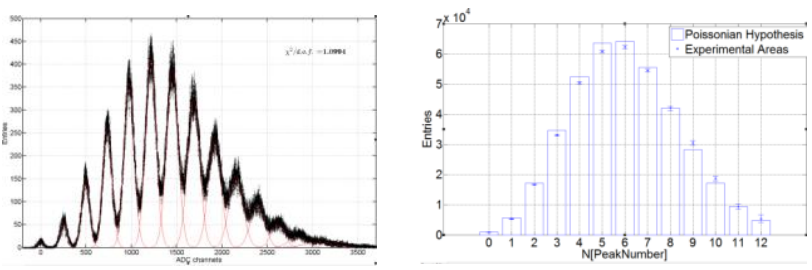

Fig.4 (a): Photoelectron spectrum probing a LED source measured with a Hamamatsu SiPM. The Individual Gaussians are shown in red. (b): Data from the light spectrum compared to a simple Poisson distribution.

\section{B. Cosmic Rays}

Another application of the CAEN kit is based on the system composed of a plastic scintillating tile directly coupled to a SiPM. The setup, shown in Fig.5, allows for cosmic rays detection and muons flux estimation. The muons, produced by the decay of pions and kaons generated by the hadronic interaction of primary cosmic rays with atmospheric nuclei, are the most common shower constituent at sea level. Cosmic muons are charged particles, produced high in the atmosphere (typically $15 \mathrm{~km}$ ) with highest penetration capability in matter. Their mass, the absence of strong interactions, their long lifetime $\left(\tau \sim 2,2 \times 10^{-6} \mathrm{~s}\right)$ and time dilatation allow muons to cross the atmosphere and reach the Earth's surface [8].

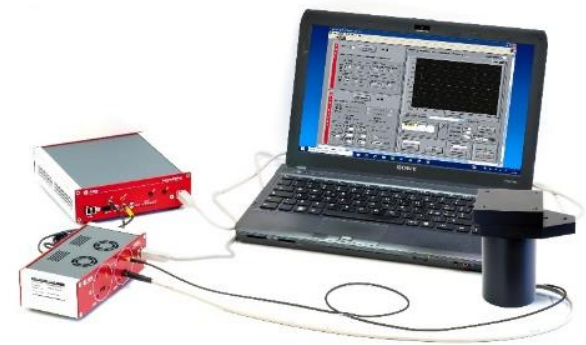

Fig.5: CAEN Educational Beta kit

The cut-off threshold of the signal produced by the system has a key role in the cosmic ray detection and it shall be set to reduce the random coincidence rate below the Hertz level and measure the cosmic rate (Fig.6).

Once the right threshold is set, the measurement of the muon vertical flux on the plastic scintillating tile can be 
performed. The students have to take into account different factors to estimate the flux: the zenith dependence of flux and the integration over the solid angle, the system geometry and the literature value corresponding to the sea level. Moreover, the detection efficiency of the system can be calculated by the comparison between the expected rate and the measured one.

The expected rate of muons across the scintillating tile is very low, requiring a fine-tuning of the system in order to achieve a significant reduction of the random count rate and enhance the system sensitivity.

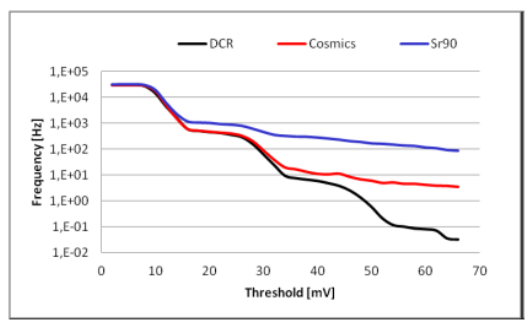

Fig.6 Signal frequency as a function of discriminator threshold. The red line represents the cosmic contribution, the black one the noise and the blue one the Sr-90 source contribution.

\section{CONCLUSIONS}

The CAEN educational kits represent the result of a strong collaboration project with university of Insubria in Italy. The proposed experiments address the essence of the phenomena, complemented by basic and advanced statistical exercises. The main goal is to inspire students, up to undergraduate physics laboratories and $\mathrm{PhD}$ courses, and guide them towards the Physics world. The aim is to encourage the use of professional electronic instrumentation, together with a new class of photon detectors and advanced statistical tools in didactic labs to perform a wide series of standard physics experiment.
[1] P. Buzhan et al., Silicon photomultiplier and its possible applications. Nuclear Instruments and Methods in Physics Research A 504, 48-52. 2003

[2] B. Dolgoshein et al., Status report on silicon photomultiplier development and its applications. Nuclear Instruments and Methods in Physics Research A 563, 368â A ,S376. 2006.

[3] D. Renker, Geiger-mode avalanche photodiodes, history, properties and problems, 2006, Nuclear
Instruments and Methods in Physics Research A, 567, 48.

[4] C. Piemonte, A new Silicon Photomultiplier structure for blue light detection, 2006, Nuclear Instruments and Methods in Physics Research A, 568, 224.

[5] http://www.caen.it/csite/CaenList.jsp?parent=378.

[6] https://www.advlab.org/event-2674088

[7] http://www.caen.it/csite/CaenFlyer.jsp?parent=424

[8] PDG July 2016, Particle Physics Booklet 